\title{
Improving multidisciplinary severe sepsis management using the Sepsis Six
}

\author{
Authors: Amar Bhat, Maryam Asghar, Gagandeep Raulia and Amit Mandal
}

Several patients in the A\&E and AMU departments are not managed optimally, which ultimately leads to increased morbidity and mortality. Many staff working in both departments were unaware of the existing trust sepsis pathway and Sepsis Six protocol for management of severe sepsis.

\section{Aims}

To assess the appropriate and timely management of these patients, and the effects on morbidity and mortality. The objectives of the audit included discussing the six steps involved in managing an acutely septic patient, analysing appropriate and timely management of patients with severe sepsis, and actively promoting teamwork to initiate the protocol.

\section{Methods}

Patients were audited prospectively over 2 months, from the middle of September to the middle of November 2014. All clerking notes of patients with a medical consultant diagnosis on post-take ward round of 'Sepsis' were analysed, and further screened for presence of severe sepsis according to national guidelines. This was re-audited prospectively from the middle of April to middle of June 2015, after one departmental and one hospital presentation, and updating of the online blood requesting system to show a 'Sepsis' tab (which would show the protocol and request appropriate bloods automatically).

\section{Results}

The initial audit demonstrated that only $1 \%$ of appropriate patients had been managed according to the existing guidelines, whereas the re-audit emphatically showed a significant improvement with $85 \%$ of eligible subjects adhering to the protocol. These patients were managed timely and appropriately with high-flow oxygen, blood cultures, lactate and urine output measurement. In the preceding audit, a mere $6 \%$ of patients were managed appropriately within the 'golden hour', with administration of fluids and antibiotics. We demonstrated an improvement to $67 \%$ on re-audit $(\mathrm{p}<0.01) .19 \%$ were admitted to ITU on initial audit (and 6\% died), which improved to
$7 \%$ on re-audit (with no deaths). Length of hospital stay was reduced from an initial audit average of 10 days to 7 days on re-audit.

\section{Conclusions}

There was significant improvement and a complete change in management of severe sepsis resulting in trust-wide updated protocols and teamwork to guide timely management, as demonstrated above, leading to a significant decrease in morbidity and mortality. 